Check for updates

Cite this: RSC Adv., 2017, 7, 36744

Received 21st June 2017

Accepted 18th July 2017

DOI: $10.1039 / \mathrm{c} 7 \mathrm{ra06909j}$

rsc.li/rsc-advances

\title{
Gold nanorods on three-dimensional nickel foam: a non-enzymatic glucose sensor with enhanced electro-catalytic performance $\uparrow$
}

\begin{abstract}
Wenqi Liu, (D) *a Xiaochun $\mathrm{Wu}^{\mathrm{b}}$ and Xiaojin Li ${ }^{\star a}$
We developed a non-enzymatic electrochemical glucose sensor with a unique three dimensional network structure by combining Ni foam with Au nanorods (Au NRs) on its surface (Au NRs/Ni). Electrochemical measurements revealed that the Au NRs/Ni foam exhibits very high sensitivity with a linear slope of 5.27 $\mathrm{mA} \mathrm{mM}-1 \mathrm{~cm}^{-2}$ for glucose detection, better than the $\mathrm{Ni}$ foam electrode and most of the reported electrodes. And its linear dynamic range is also broader than the Ni foam electrode. Moreover, upon light excitation of the localized surface plasmon resonance (SPR) of the Au NRs, light-enhanced electrochemical activities are observed from the Au NRs/Ni foam, suggesting the possibility of modifying electrochemical sensors by surface plasmon resonance. These results highlight the potentials of the $\mathrm{Au}$ NRs/Ni foam electrode in practical glucose monitoring and plasmonic effects in photoelectrochemical sensing
\end{abstract}

\section{Introduction}

Extensive studies have been devoted to the development of glucose sensors in the past few decades due to their considerable applications in the diagnosis of diabetes, waste water treatment and food analysis. ${ }^{1}$ Basically, there are two categories of glucose sensors: enzymatic and non-enzymatic ones. ${ }^{2}$ Although enzymatic detection provides high sensitivity and good selectivity, its application is restricted due to the intrinsic nature of enzymes including their instability and sensitivity to their environment. ${ }^{3}$ Therefore, tremendous efforts have been directed toward the improvement of non-enzymatic glucose (NEG) sensors. ${ }^{4,5}$ Electrochemical method is often employed for NEG detection, which is based on the current response of glucose oxidation at the electrode surface modified by specific materials. Although the mechanism of glucose oxidation at the electrode surface is still not fully understood, a variety of materials including noble metals, ${ }^{6}$ metal oxides/hydroxides, ${ }^{7}$ carbon nanotubes, ${ }^{8}$ graphene, ${ }^{9}$ polymers, and hybrids ${ }^{10}$ have been explored and applied to NEG sensors.

Among numerous materials, noble metal nanoparticles (NMNPs), such as Au, Ag, Pt, Pd, exhibit high electrocatalytic activities, long-term stability and good biocompatibility ${ }^{11,12}$ and therefore have been extensively explored as electrode materials

${ }^{a}$ Qingdao Institute of Bioenergy and Bioprocess Technology, Chinese Academy of Sciences, Qingdao 266101, P. R. China. E-mail: liuwq@qibebt.ac.cn

${ }^{b}$ CAS Key Laboratory of Standardization and Measurement for Nanotechnology, National Center for Nanoscience and Technology, Beijing 100190, P. R. China

$\dagger$ Electronic supplementary information (ESI) available. See DOI: 10.1039/c7ra06909j for glucose oxidation. Furthermore, NMNPs display unique optical properties, especially for $\mathrm{Au}, \mathrm{Ag}$ nanoparticles, which possess strong extinctions in the visible and NIR spectral region owing to localized surface plasmon resonances (LSPR). ${ }^{13,14}$ NMNPs can act as plasmon catalysts or photosensitizers by transferring some of the absorbed plasmonic energy through either resonant energy transfer or direct hot-electron injection to the reactants, thereby improving their own catalytic activities or performances of attached substrates. ${ }^{15-17}$ This light-enhanced activity has recently attracted great attention in solar harvesting field. ${ }^{18}$

Encouraged by the achievements based on plasmon enhanced catalytic activities, we hereby propose a novel sensing platform that can utilize LSPR to enhance the glucose oxidation and thus to improve the sensitivity of the glucose sensor. The platform consists of Au nanorods (Au NRs)-modified Ni foam (Au NRs/Ni). $\mathrm{Au}$ NRs were employed as plasmon catalysts due to their aspect ratio-dependent LSPR responses and well-developed synthesis method. ${ }^{19} \mathrm{Ni}$ foam was chosen as the support for the Au NRs due to its high conductivity, unique three dimensional network structure, large loading amount of active materials and low price. ${ }^{20,21}$ In addition, Ni foam can directly act as a sensing platform for glucose detection in alkaline solution, which is mediated by $\mathrm{Ni}(\mathrm{II}) / \mathrm{Ni}(\mathrm{III})$ redox couple. ${ }^{22}$ At first, using this nanoplatform, we investigated its electrochemical sensing of glucose in the absence of plasmon excitation by cyclic voltammetry and chronoamperometry. Significantly enhanced electrocatalytic performance has been observed using the Au NRs/Ni electrode for glucose oxidation. And it shows higher sensitivity and broader linear range than the $\mathrm{Ni}$ foam electrode. Upon plasmon excitation with simulated sunlight irradiation, light- 
enhanced electrochemical sensing of glucose is exhibited, indicating the feasibility of further improvements in sensitivity, dynamic range, and detection limit and highlighting the potential applications of plasmon effects for electrochemical sensing.

\section{Experimental}

\section{Materials}

Sodium borohydride $\left(\mathrm{NaBH}_{4}\right)$, cetyltrimethylammonium bromide (CTAB), chlorauric acid $\left(\mathrm{HAuCl}_{4} \cdot 3 \mathrm{H}_{2} \mathrm{O}\right)$, silver nitrate $\left(\mathrm{AgNO}_{3}\right)$, and L-ascorbic acid (AA) were purchased from Alfa Aesar and used as received. Ni foams (110 PPI, $320 \mathrm{~g} \mathrm{~m}^{-2}, 1 \mathrm{~mm}$ in thickness) were purchased from Changsha Lyrun New Material Co. Ltd., China. Other reagents and chemicals were at least analytical reagent grade and purchased from Beijing Chemical Reagent Company (Beijing, China). Milli-Q water (18 $\mathrm{M} \Omega \mathrm{cm}$ ) was used for all solution preparations.

\section{Instruments}

UV-vis-NIR absorption spectra were recorded on a Hitachi U4100. X-ray diffraction (XRD) patterns were acquired using a Rigaku MiniFlex X-ray diffractometer equipped with $\mathrm{Cu} \mathrm{K} \alpha$ radiation source operated at $50 \mathrm{kV} / 300 \mathrm{~mA}$. Transmission electron microscopy (TEM) and scanning electron microscopy (SEM) images were obtained with a TecnaiG2 20 S-TWIN operating at an acceleration voltage of $200 \mathrm{kV}$ and a Hitachi S-4800 equipped with energy dispersive spectroscopy (EDS) operating at an acceleration voltage of $10 \mathrm{kV}$, respectively. Electrochemical measurements were performed on a CHI 650 electrochemical analyzer (Shanghai, China).

\section{Synthesis of Au NRs}

$\mathrm{Au}$ NRs were synthesized through the seed-mediated growth method. CTAB-capped $\mathrm{Au}$ seeds were prepared as follows: 7.5 $\mathrm{mL}$ of $0.1 \mathrm{M}$ CTAB solution was mixed with $100 \mu \mathrm{L}$ of $25 \mathrm{mM}$ $\mathrm{HAuCl}_{4}$ and deionized water to $9.4 \mathrm{~mL}$. Then, ice-cold $\mathrm{NaBH}_{4}$ $(0.6 \mathrm{~mL}, 0.01 \mathrm{M})$ was added under vigorous stirring. The seed solution was then kept at room temperature and used within 2$5 \mathrm{~h}$. Then, Au NRs were synthesized by reduction of $\mathrm{HAuCl}_{4}$ with AA on Au seeds: $120 \mu \mathrm{L}$ seed solution was added to the growth solution consisted of CTAB (100 mL, $0.1 \mathrm{M}), \mathrm{HAuCl}_{4}(2 \mathrm{~mL}, 25$ $\mathrm{mM}), \mathrm{AgNO}_{3}(1.05 \mathrm{~mL}, 10 \mathrm{mM})$, and $\mathrm{AA}(552 \mu \mathrm{L}, 0.1 \mathrm{M})$ to initiate the growth. The resultant reaction solution was gently mixed by inversion and then left undisturbed. After $12 \mathrm{~h}, \mathrm{AA}$ $(0.1 \mathrm{M}, 55.2 \mu \mathrm{L})$ was added twice with $40 \mathrm{~min}$ interval. After about $3 \mathrm{~h}$, the Au NRs were separated from the growth solution by centrifugation (12 $000 \mathrm{rpm}$ for $5 \mathrm{~min}$ ) twice. The precipitation was collected and redispersed in $20 \mathrm{~mL}$ of deionized water.

\section{Preparation of Au NRs/Ni foam electrode}

$1 \mathrm{~cm} \times 1 \mathrm{~cm} \times 1 \mathrm{~mm}$ Ni foam was washed with acetone, etched in $0.1 \mathrm{M} \mathrm{HCl}$ for $1 \mathrm{~h}$ to remove the thin layer of oxide, and rinsed with ultrapure water prior to use. For the modification of the electrode, $200 \mu \mathrm{L}$ of the synthesized Au NRs $(0.39 \mu \mathrm{mol} \mathrm{Au}$ atoms, calculated by Fig. S1 $\dagger$ ) was added onto the Ni foam surface. The electrode was then dried under infrared lamp.

\section{Preparation of Au NRs/glassy carbon electrode (GCE)}

$\operatorname{GCE}(\Phi=4 \mathrm{~mm})$ was polished with $1.0,0.3$, and $0.05 \mu \mathrm{m}$ $\alpha$-alumina powders in sequence, and rinsed thoroughly with deionized water between each polishing step, then sonicated in $1: 1 \mathrm{HNO}_{3} /$ ethanol and deionized water, and dried at room temperature prior to modification. A volume of $7.5 \mu \mathrm{L}$ of Nafion $(5 \% \mathrm{v} / \mathrm{v}$, in alcohol) was mixed with $100 \mu \mathrm{L}$ of the synthesized $\mathrm{Au}$ NRs, then $7 \mu \mathrm{L}$ of the resulting solution was applied to the GCE and allowed to dry for $1 \mathrm{~h}$.

\section{Electrochemical measurements}

All of electrochemical analyses were carried out with a three electrode system, in which a platinum wire and a $\mathrm{Ag} / \mathrm{AgCl}$ (sat. $\mathrm{KCl})$ electrode were used for counter electrode and reference electrode, respectively. Ni foam, Au NRs/Ni foam, and Au NRs/ GCE act as the working electrode. All electrochemical measurements were performed on a CHI 650 electrochemical analyzer (Shanghai, China) at ambient temperature. The scan rate range in cyclic voltammetry (CV) was $0.001 \mathrm{~V} \mathrm{~s}^{-1}$ to $1 \mathrm{~V} \mathrm{~s}^{-1}$. The optimal working potential was set to $+0.600 \mathrm{~V}$ for measurements of glucose. CV measurements were performed in a glucose concentration range of 0-5 $\mathrm{mM}$. Current densities were normalized to the geometrical area of the working electrode. All potentials were referred to the reference electrode unless specified.

\section{Results and discussion}

The synthesis of Au NRs was simple and efficient through the seed-mediated growth method. ${ }^{19}$ The obtained Au NRs were characterized by TEM and UV-vis-NIR extinction spectra. As shown in Fig. 1, their longitudinal SPR peaks were located at $700 \mathrm{~nm}$, and the size distributions were narrow as determined from the TEM images, with an average length of $43.1 \mathrm{~nm}$ and average width of $15.1 \mathrm{~nm}$ (Table S1 $\dagger$ ). SEM images for the Ni foam substrate at various magnifications are shown in Fig. 2A$\mathrm{D}$, displaying a $3 \mathrm{D}$, highly porous and cross-linked structure. Fig. 2a-d show the SEM images after the obtained Au NRs were concentrated and deposited onto the pretreated $\mathrm{Ni}$ foam. It can be seen through Fig. $2 \mathrm{C}$ and $\mathrm{c}$ that the Ni foam surface is covered with Au NRs. Comparing Fig. 2D with Fig. 2d, it is easily seen that the $\mathrm{Au}$ NRs deposition results in a significant morphology change of the Ni foam surface, demonstrating the efficient formation of $\mathrm{Au}$ NRs aggregates. The elemental analysis of the as-prepared samples was further performed using EDS. EDS element mappings of $\mathrm{Au}$ and $\mathrm{Ni}$ obtained from the SEM images of Au NRs/Ni foam is shown in Fig. 2E. The amount of $\mathrm{Au} \mathrm{NRs}$ on the $\mathrm{Ni}$ foam can be controlled by varying the dropping amount or dropping times.

The chemical composition and crystal phase of the samples were further examined by XRD analysis. The Ni foam shows three sharp diffraction peaks at $2 \theta=44.5^{\circ}, 51.8^{\circ}$, and $76.3^{\circ}$ (Fig. 2F, black line), which match (111), (200) and (220) planes of the face centered cubic (fcc) phase of Ni metal (JCPDS card no. 65-2865), respectively. After modification with Au NRs, new diffraction peaks appearing in the XRD pattern (Fig. 2F, red line) at $2 \theta=38.2^{\circ}$ and $64.5^{\circ}$, which can be well indexed to the 
A

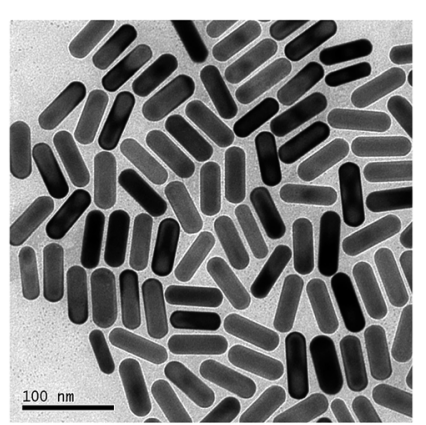

B

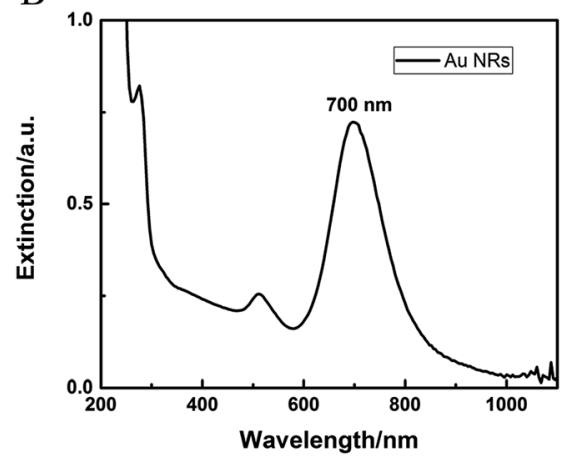

Fig. 1 TEM images (A) and UV-vis-NIR extinction spectra (B) of the obtained Au NRs.
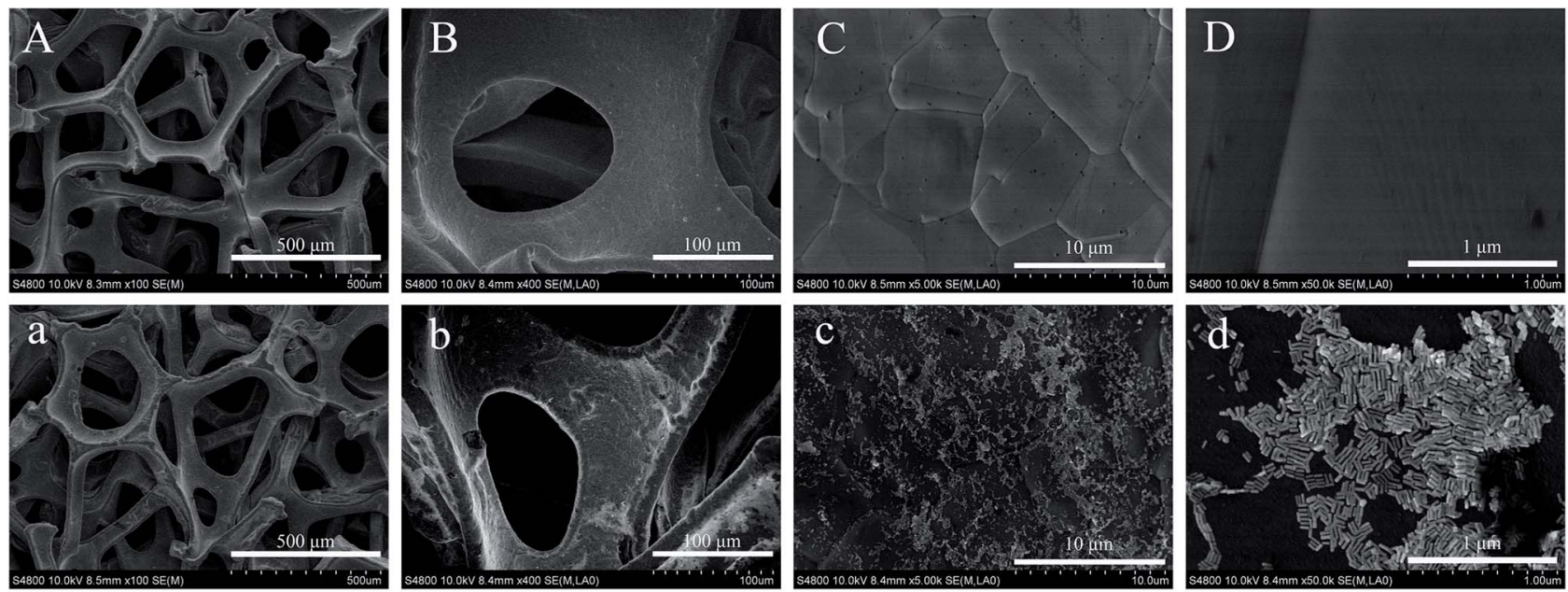

$\mathrm{E}$
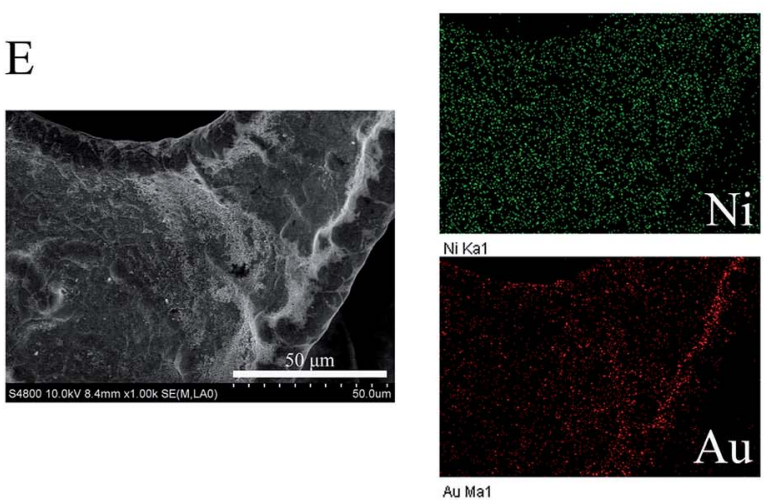

$\mathrm{F}$

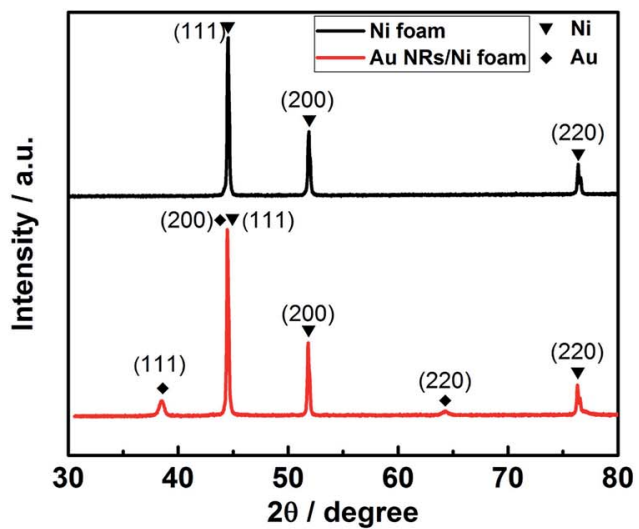

Fig. 2 SEM images of $\mathrm{Ni}$ foam substrate (A to D) and Au NRs/Ni foam electrode (a to d); (E) SEM image of Au NRs/Ni foam and EDS element mappings of $\mathrm{Ni}$ and $\mathrm{Au}$ atoms; (F) XRD patterns of Ni foam substrate and Au NRs/Ni foam electrode.

(111) and (220) planes of fcc Au (JCPDS card no. 65-2870). In addition, the fcc Ni (111) peak almost overlaps with the fcc $\mathrm{Au}$ (200) peak. The above XRD results demonstrate that the Au NRs were modified on the Ni foam surface. The resulting electrode was then characterized by cyclic voltammetry (CV) in detail.

For evaluating the electrochemical properties of Au NRs modified Ni foam electrode, cyclic voltammograms of the nickel foam substrate, Au NRs/GCE and Au NRs/Ni foam electrode were carried out in $0.1 \mathrm{M} \mathrm{KOH}$ at a scan rate of $100 \mathrm{mV} \mathrm{s}^{-1}$ (Fig. 3). The CVs are similar to those reported in the literature for $\mathrm{Ni}$ and $\mathrm{Au}$ electrode in alkaline solution. ${ }^{23,24}$ As can be seen, four redox peaks, A/B, a/b, C/D, and c/d are present. The a/ b couple is due to the $\mathrm{Ni}(\mathrm{II}) / \mathrm{Ni}$ (III) redox couple, the same to the $\mathrm{A} / \mathrm{B}$ couple. While $\mathrm{C} / \mathrm{D}$ and $\mathrm{c} / \mathrm{d}$ process is related to gold oxide formation and subsequent reduction to Au0. ${ }^{25}$ The voltammetric profile of the modified electrode is the weighted sum 


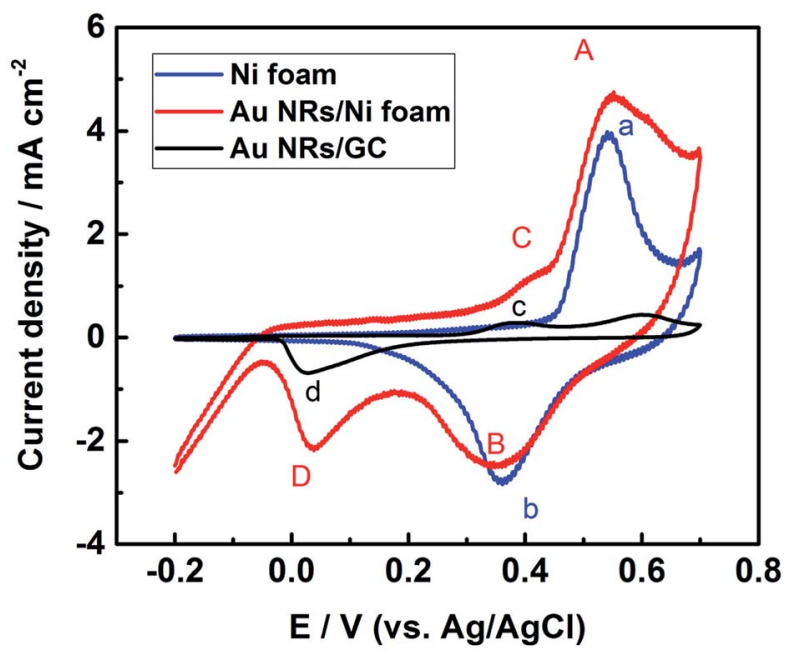

Fig. 3 Cyclic voltammograms of the nickel foam substrate, the $A u$ $\mathrm{NRs} / \mathrm{Ni}$ foam electrode, and the Au NRs/GCE measured in $0.1 \mathrm{M} \mathrm{KOH}$ at a scan rate of $100 \mathrm{mV} \mathrm{s}^{-1}$.

of the electrochemical behaviours of $\mathrm{Au}$ and $\mathrm{Ni}$ catalyst. In addition, the Au-Ni composite electrode exhibits good stability in alkaline solutions (Fig. S2†), possibly due to the electrostatic binding between the positively charged Au NRs and negatively charged Ni foam surface. ${ }^{26}$ Moreover, the current density for the oxidation/reduction of Au NRs/Ni foam electrode is higher than that of Ni foam substrate.

The electrochemical behaviors of glucose oxidation were investigated by CV. Fig. $4 \mathrm{~A}$ and $\mathrm{B}$ and $\mathrm{S} 3 \uparrow$ display the CVs over a voltage range from -0.2 to $0.7 \mathrm{~V}$ for the nickel foam substrate, $\mathrm{Au}$ NRs/Ni foam electrode, and Au NRs/GCE in $0.1 \mathrm{M} \mathrm{KOH}$ solution with different glucose concentrations at a sweep rate of $100 \mathrm{mV} \mathrm{s}^{-1}$. As Fig. 4A shows, one redox peak is observed at the $\mathrm{Ni}$ foam due to the glucose oxidation and reduction mediated by $\mathrm{Ni}(\mathrm{II}) / \mathrm{Ni}$ (III) redox couple. The oxidation currents at $0.4-0.6 \mathrm{~V}$ increase gradually with the introduction of glucose from 0 to
$1 \mathrm{mM}$, indicating the electrochemical oxidation of glucose, but the currents gradually become saturated from 1 to $5 \mathrm{mM}$. Fig. $\mathrm{S} 3 \dagger$ and $4 \mathrm{~B}$ shows the CVs for Au NRs/GCE and Au NRs/Ni foam electrode with different glucose concentrations. They exhibit some common properties. In the positive potential scan two oxidation peaks appear both in Fig. S3 $\uparrow$ and $4 \mathrm{~B}$ at about $+0.45 \mathrm{~V}$ (peak A) and $+0.65 \mathrm{~V}$ (peak B), peak $\mathrm{A}$ is due to the direct electrochemical oxidation of glucose by the electroactive species of $\mathrm{Au}(\mathrm{OH})$ ads and peak B is attributed to the further oxidation of gluconolactone generated from glucose oxidation (peak A). While during the reverse scan a single oxidation peak at about $0 \mathrm{~V}$ (peak C) is also observed both in Fig. S3† and 4B. As Fig. $\mathrm{S} 3 \dagger$ and $4 \mathrm{~B}$ show, the rate of oxidation increases greatly and a welldefined peak (peak C) is soon developed. The formation of peak $\mathrm{C}$ is due to the re-oxidation of glucose for it's in the same potential region as soon as the Au oxides are reduced. ${ }^{27}$ So peak $\mathrm{C}$ reflects the oxidation of glucose by Au oxides. In addition, the difference between Fig. S3 $\uparrow$ and $4 \mathrm{~B}$ is that no glucose oxidation and reduction peaks mediated by $\mathrm{Ni}($ III)/Ni(III) redox couple are found in Fig. S3. $\dagger$ Furthermore, the peak current for Au NRs/Ni foam electrode is higher than that of $\mathrm{Ni}$ foam substrate, and proportional to the glucose concentration up to $5 \mathrm{mM}$, since more electrons could be provided with a high content of reaction reagents during electrochemical oxidation for $\mathrm{Au} \mathrm{NRs} / \mathrm{Ni}$ foam electrode.

To further evaluate the sensitivity of the NEG sensor based on $\mathrm{Au} \mathrm{NRs/Ni} \mathrm{foam} \mathrm{electrocatalyst,} \mathrm{the} \mathrm{chronoamperometry} \mathrm{is}$ performed. Fig. 5A depicts the amperometric response curves of the nickel foam substrate and Au NRs/Ni foam electrode upon the successive addition of glucose at $0.6 \mathrm{~V} v s$. $\mathrm{Ag} / \mathrm{AgCl}$ electrode. The currents increase immediately with the increase of glucose concentrations, indicating the sensitive and rapid responses as a glucose monitor. And the Au NRs/Ni foam electrode shows larger current response toward glucose sensing than the nickel foam. Fig. 5B displays the linear relationship between the current density and the glucose concentrations. The sensitivity of the sensors could be calculated from the linear regression
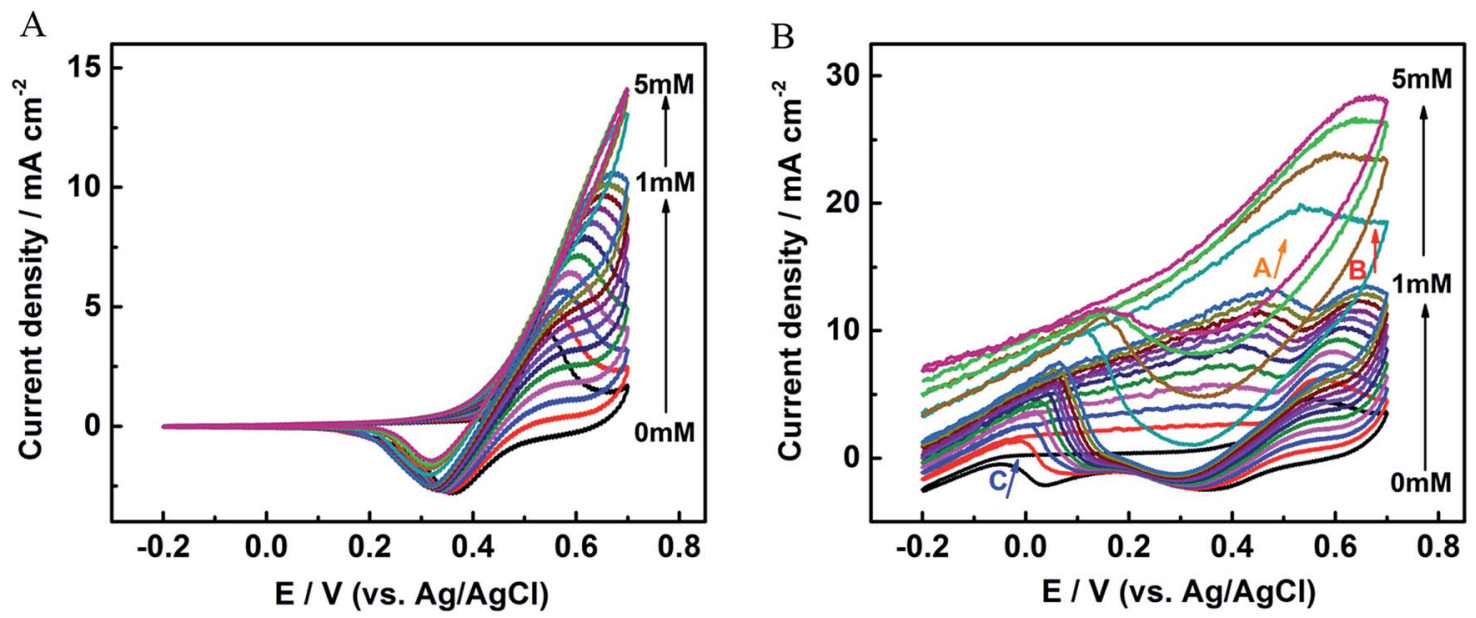

Fig. $4 \mathrm{CV}$ curves of (A) the nickel foam substrate and (B) the Au NRs/Ni foam electrode in a series of glucose concentration measured in $0.1 \mathrm{M}$ $\mathrm{KOH}$ at a scan rate of $100 \mathrm{mV} \mathrm{s}^{-1}$. 
A

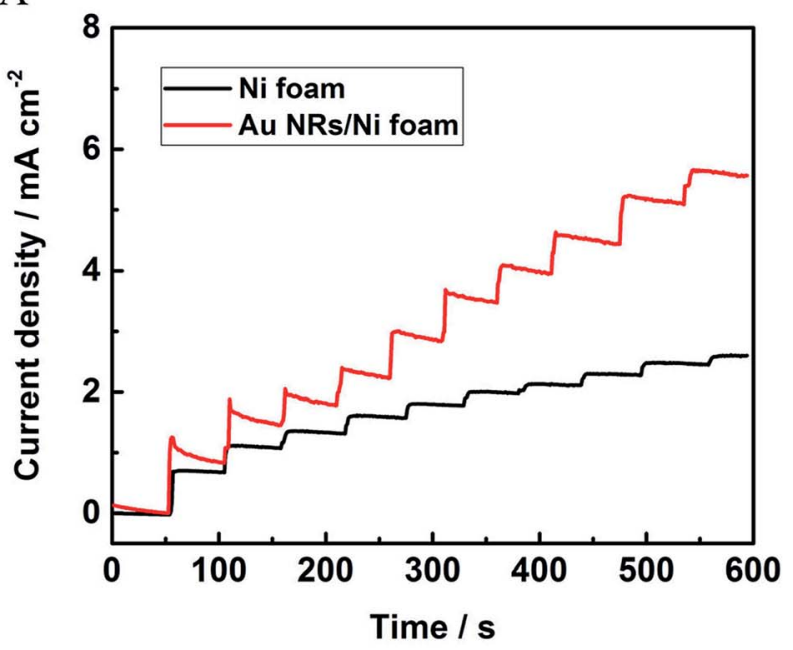

B

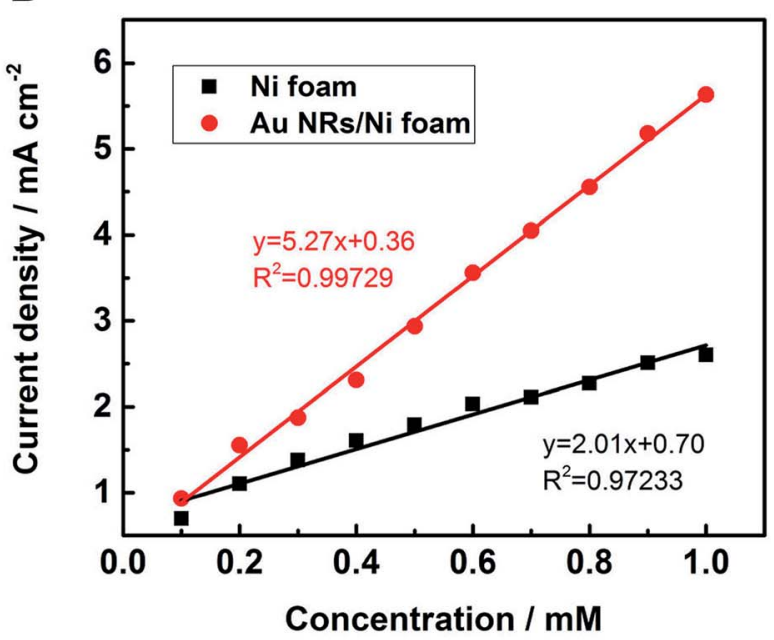

Fig. 5 (A) Amperometric response curves and (B) the corresponding calibration curves of the nickel foam substrate and the Au NRs/Ni foam electrode upon successive addition of glucose at $0.6 \mathrm{~V}$ (vs. $\mathrm{Ag} / \mathrm{AgCl}$ ).

equations. The sensitivity of Au NRs/Ni foam is $5.27 \mathrm{~mA} \mathrm{mM}^{-1}$ $\mathrm{cm}^{-2}$ for glucose detection, which is 2.62 times as high as the nickel foam $\left(2.01 \mathrm{~mA} \mathrm{mM}{ }^{-1} \mathrm{~cm}^{-2}\right)$. Table S2† summarizes the sensitivity of several typical NEG sensors with different electrodes reported previously. It should be noted that the Au NRs/ $\mathrm{Ni}$ foam electrode possesses excellent sensitivity toward glucose detection among these electrodes. And the limit of detection can be calculated to be $14 \mu \mathrm{M}$ for the Au NRs/Ni foam electrode based on the signal-to-noise ratio of $3(\mathrm{~S} / \mathrm{N}=3)$. The excellent sensitivity of $\mathrm{Au} \mathrm{NRs} / \mathrm{Ni}$ foam can be attributed to the high electrocatalytic activity of Au NRs. Moreover, the well-distributed $\mathrm{Au}$ NRs on the surface of $\mathrm{Ni}$ foam favor the full access of single $\mathrm{Au}$ NR to glucose, which significantly enhances the electrochemical response toward glucose oxidation. The amperometric response curve of the Au NRs/GCE as control is shown in Fig. S4, $\dagger$ which indicates that no $\mathrm{Ni}$ foam as the substrate significantly decreased the sensitivity.

The photoelectrochemical properties of $\mathrm{Au}$ NRs/Ni foam electrode were studied under simulated sunlight irradiation at room temperature in $0.1 \mathrm{M} \mathrm{KOH}$ solution. Fig. 6A shows the CVs over a voltage range from -0.2 to $0.7 \mathrm{~V}$ for the Au NRs/Ni foam electrode with light on/off. The CV curves were measured upon successive addition of $0.1 \mathrm{mM}$ glucose between every two scans. Light was turned on or off just before adding glucose. As can be seen, a clear increase in current change between two scans is observed when the light is switched on, and the current change
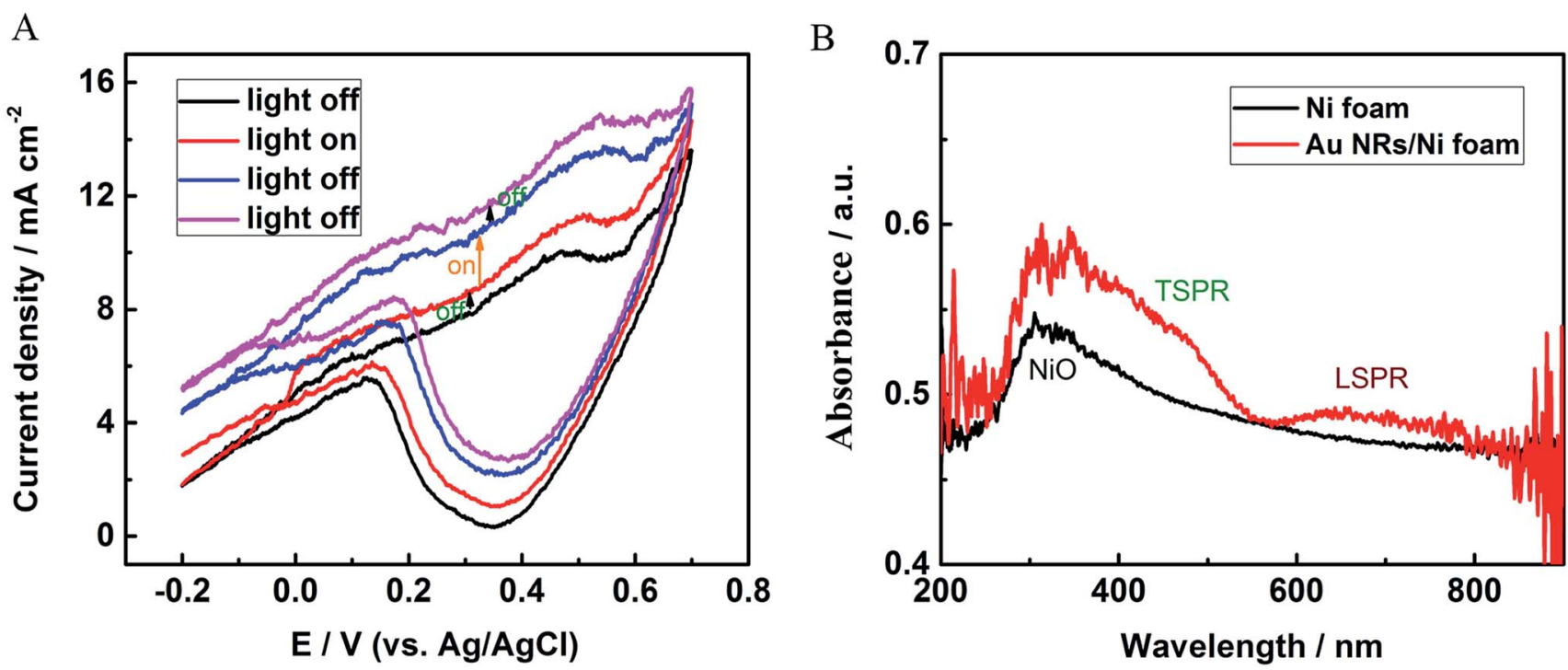

Fig. 6 Plasmon enhanced electrochemical oxidation of glucose: (A) CV curves of the Au NRs/Ni foam electrode measured with light on/off in $0.1 \mathrm{M} \mathrm{KOH}$ at a scan rate of $100 \mathrm{mV} \mathrm{s}^{-1}, 0.1 \mathrm{mM}$ glucose was added between two scans; (B) UV-vis-NIR spectra of the nickel foam substrate and the Au NRs/Ni foam electrode. 
immediately returned to its initial state after the light is turned off, suggesting the enhanced redox reactions on the sensor surface under sun light illumination. By statistics, the current change with light on is 1.77 times of the current change with light off, and the percentage of the photo-enhanced effect is about $44 \%$ in the total signal. The UV-vis-NIR spectra were measured to prove light absorption by Au NRs/Ni foam electrode. As Fig. 6B shows, Ni foam presents an absorption band at about $350 \mathrm{~nm}$, which is ascribed to the absorption of $\mathrm{NiO} .^{28}$ In contrast, two additional broad absorption bands are found for the $\mathrm{Au}$ NRs/Ni foam between 500 and $700 \mathrm{~nm}$, which correspond to the transverse and longitudinal SPR bands of the Au NRs, respectively. Band shifting and broadening are caused by the assembly of Au NRs on Ni foam. As the ultraviolet part of simulated sunlight irradiation is low, the effect of $\mathrm{NiO}$ absorption on light-enhanced electrochemical responses can be ignored. In contrast, plasmonic photocatalysis of the Au NRs is the main cause of improved photoelectrochemical responses for glucose. In addition, whether the Au NRs act as the photosensitizers and lead to extra photocurrent increase through transferring plasmonic energy to underneath $\mathrm{Ni}$ foam need further investigations. In all, our results show that the plasmonic effect has a great application potential in photoelectric sensors.

\section{Conclusions}

In summary, we successfully developed a non-enzymatic electrochemical glucose sensor by deposition of $\mathrm{Au}$ nanorods on $\mathrm{Ni}$ foam surface. The deposition of dense and well-dispersed $\mathrm{Au}$ NRs on the electrodes could significantly promote the electrocatalytic ability toward the oxidation of glucose during the electrochemical tests. The Au NRs/Ni foam shows better sensitivity among the reported glucose electrodes with $5.27 \mathrm{~mA}$ $\mathrm{mM}^{-1} \mathrm{~cm}^{-2}$ for glucose detection and provides larger linear dynamic range than Ni foam electrode. These results confirm that $\mathrm{Au}$ nanostructures could work as excellent electrode materials for the sensing of glucose. Furthermore, Au NRs/Ni foam shows absorptions in the visible spectrum due to the localized SPR, and thus can effectively enhance the glucose oxidation through light excitation of plasmonic bands and further improve the sensitivity. We believe that the proposed glucose sensor shows great promises for applications in practical glucose monitoring. And the plasmon enhanced electrocatalytic performance can also be extended to other reactions. Further research is in progress.

\section{Acknowledgements}

This work was financially supported by the Key Program for International S\&T Cooperation Projects of China (2016YFE0125700) and the Postdoctoral Application Research Project of Qingdao City.

\section{Notes and references}

1 Q. Wu, L. Wang, H. Yu, J. Wang and Z. Chen, Chem. Rev., 2011, 111, 7855.
2 K. E. Toghill and R. G. Compton, Int. J. Electrochem. Sci., 2010, 5, 1246.

3 R. Wilson and A. P. F. Turner, Biosens. Bioelectron., 1992, 7, 165.

4 G. F. Wang, X. P. He, L. L. Wang, A. X. Gu, Y. Huang, B. Fang, B. Y. Geng and X. J. Zhang, Microchim. Acta, 2013, 180, 161.

5 S. Y. Tee, C. P. Teng and E. Ye, Mater. Sci. Eng. C, 2017, 70, 1018.

6 S. H. Kim, J. B. Choi, Q. N. Nguyen, J. M. Lee, S. Park, T. D. Chung and J. Y. Byun, Phys. Chem. Chem. Phys., 2013, 15, 5782.

7 F. Huang, Y. Zhong, J. Chen, S. Li, Y. Li, F. Wang and S. Feng, Anal. Methods, 2013, 5, 3050.

8 N. Q. Dung, D. Patil, H. Jung and D. Kim, Biosens. Bioelectron., 2013, 42280.

9 L. Luo, L. Zhu and Z. Wang, Bioelectrochemistry, 2012, 88, 156.

10 N. Hui, S. Wang, H. Xie, S. Xu, S. Niu and X. Luo, Sens. Actuators, B, 2015, 221, 606.

11 M. M. Biener, J. Biener, A. Wichmann, A. Wittstock, T. F. Baumann, M. Baumer and A. V. Hamza, Nano Lett., 2011, 11, 3085.

12 J. Biener, A. Wittstock, L. A. Zepeda-Ruiz, M. M. Biener, V. Zielasek, D. Kramer, R. N. Viswanath, J. Weissmuller, M. Baumer and A. V. Hamza, Nat. Mater., 2009, 8, 47.

13 C. J. Murphy, T. K. San, A. M. Gole, et al., J. Phys. Chem. B, 2005, 109, 13857.

14 Y. Xia, X. Xia and H. C. Peng, J. Am. Chem. Soc., 2015, 137, 7947.

15 Z. Zheng, T. Tachikawa and T. Majima, J. Am. Chem. Soc., 2015, 137, 948.

16 R. Jiang, F. Qin, Q. Ruan, et al., Adv. Funct. Mater., 2014, 24, 7328.

17 F. Wang, C. Li, H. Chen, et al., J. Am. Chem. Soc., 2013, 135, 5588.

18 R. B. Jiang, B. X. Li, C. H. Fang and J. F. Wang, Adv. Mater., 2014, 26, 5274.

19 N. R. Jana, L. Gearheart and C. J. Murphy, Adv. Mater., 2001, 13, 1389.

20 W. Q. Yang, S. H. Yang, W. Sun, G. Q. Sun and Q. Xin, Electrochim. Acta, 2006, 52, 9.

21 Y. L. Wang, Y. Q. Zhao, C. L. Xu, D. D. Zhao, M. W. Xu, Z. X. Su and H. L. Li, J. Power Sources, 2010, 195, 6496.

22 M. Fleischmann, K. Korinek and D. Pletcher, J. Electroanal. Chem. Interfacial Electrochem., 1971, 31, 39.

23 W. Visscher and E. Barendrecht, J. Electroanal. Chem., 1983, 154, 69.

24 M. Bektiwaska-Brzezinska, Electrochim. Acta, 1985, 30, 1193. 25 S. Bruckenstein and M. Shay, J. Electroanal. Chem., 1985, 188, 131.

26 N. Hernández, R. Moreno, A. J. Sánchez-Herencia and J. L. G. Fierro, J. Phys. Chem. B, 2005, 109, 4470.

27 I. G. Casella, M. R. Guascito and T. R. I. Cataldi, Anal. Chim. Acta, 1999, 398, 153.

28 F. Farzaneh and S. Haghshenas, Mater. Sci. Appl., 2012, 3, 697. 